\title{
Absolute and relative QPCR quantification of plasmid copy number in Escherichia coli
}

\author{
Changsoo Lee, Jaai Kim, Seung Gu Shin, Seokhwan Hwang* \\ School of Environmental Science and Engineering, Pohang University of Science and Technology, San 31 \\ Hyoja-dong, Nam-gu, Pohang, Kyungbuk 790-784, Republic of Korea
}

Received 31 May 2005; received in revised form 28 August 2005; accepted 10 November 2005

\begin{abstract}
Real-time QPCR based methods for determination of plasmid copy number in recombinant Escherichia coli cultures are presented. Two compatible methods based on absolute and relative analyses were tested with recombinant $E$. coli DH5 $\alpha$ harboring pBR322, which is a common bacterial cloning vector. The separate detection of the plasmid and the host chromosomal DNA was achieved using two separate primer sets, specific for the plasmid $\beta$-lactamase gene (bla) and for the chromosomal D-1deoxyxylulose 5-phosphate synthase gene $(d x s)$, respectively. Since both bla and $d x s$ are single-copy genes of pBR322 and $E$. coli chromosomal DNA, respectively, the plasmid copy number can be determined as the copy ratio of bla to $d x s$.

These methods were successfully applied to determine the plasmid copy number of pBR322 of E. coli host cells. The results of the absolute and relative analyses were identical and highly reproducible with coefficient of variation (CV) values of 2.8-3.9\% and $4.7-5.4 \%$, respectively. The results corresponded to the previously reported values of pBR322 copy number within $E$. coli host cells, 15-20.

The methods introduced in this study are convenient to perform and cost-effective compared to the traditionally used Southern blot method. The primer sets designed in this study can be used to determine plasmid copy number of any recombinant $E$. coli with a plasmid vector having bla gene.
\end{abstract}

() 2005 Elsevier B.V. All rights reserved.

Keywords: Plasmid copy number; Real-time quantitative PCR (QPCR); Absolute quantification; Relative quantification; pBR322; Escherichia coli

\section{Introduction}

Plasmid is frequently used as a vehicle to carry foreign genes into a bacterial host cell. Recombinant gene

\footnotetext{
* Corresponding author. Tel.: +82 542792282 ; fax: +82 542798299 .

E-mail address: shwang@ postech.ac.kr (S. Hwang).
}

expression systems using bacterial plasmids are widely used to produce many types of valuable proteins, such as therapeutics and vaccines. Plasmid stability is essential in fermentation with recombinant systems for the maintenance and expression of recombinant genes (Argyropoulos and Savva, 1997). Therefore, careful assessment of plasmid stability is necessary for consistent production of target products. Plasmid stability 
also provides an insight into understanding general features of the cells during replication, transcription and translation in the presence of extrachromosomal DNA (Summers, 1991).

Genetic stability of recombinant plasmid can be evaluated both qualitatively and quantitatively (Fujimura et al., 1996). Qualitatively, it should be shown that the structure of the inserted gene is stable. Qualitative analysis is generally easy to perform because bacterial plasmids is readily recovered and propagated in host cells. Quantitatively, it should be demonstrated that the copy number of the recombinant plasmid is stable within the host cells. Therefore, a reliable method to quantify the copy number of the plasmid of interest is needed for quantitative analysis.

A plasmid copy number ( $\mathrm{PCN}$ ) assay is a widely used method in quantitative genetic stability analysis (Fujimura et al., 1996). Traditionally, the plasmid copy number has been determined by measuring band density produced through a DNA hybridization method (Korpela et al., 1987; Fujimura et al., 1996). However, the method presents safety problems in handling radioactively labeled probes, and the procedure is laborious and time-consuming (Pushnova et al., 2000). The radioactive probe used for hybridization can also cause inaccuracy of quantification results due to its short life span (Pushnova et al., 2000). Therefore, a reliable method is required for accurate determination of plasmid copy number.

Real-time quantitative PCR (QPCR) technology offers fast and reliable quantification of any target sequence in a sample (Burgos et al., 2002). While there are many methods available for quantification of nucleic acids, real-time QPCR is at present the most sensitive and precise method (Ferre, 1992; Klein, 2002). In this study, we introduced two convenient ways to determine the plasmid copy number: absolute and relative quantification methods using a real-time QPCR. Adequacy of each method was also demonstrated by determining the plasmid copy number of the pBR322 within Escherichia coli DH5 $\alpha$. To our knowledge, this is the first report to determine plasmid copy number by absolute and relative quantification methods using a real-time QPCR. The plasmid and the host cell are most commonly used. These methods are faster and cost-effective compared to the DNA hybridization without any safety problems. Moreover, the methods are easy to perform and thus could be applied to mon- itor plasmid copy number in a time-course study or in a recombinant bioprocess.

\section{Materials and methods}

\subsection{Strains}

E. coli $\mathrm{DH} 5 \alpha$, grown in $\mathrm{LB}$ medium at $37^{\circ} \mathrm{C}$, was used as the host strain for all transformation procedures. Recombinant $E$. coli transformed using pBR322 was cultured in $\mathrm{LB}$ medium at $37^{\circ} \mathrm{C}$ with addition of $100 \mu \mathrm{g} / \mathrm{ml}$ of ampicillin (Sigma). The recombinant strain was used as a test organism for the determination of plasmid copy number.

\subsection{Design of primer sets for real-time $Q P C R$}

Two primer sets specific to the $\beta$-lactamase gene (bla) and to the D-1-deoxyxylulose 5-phosphate synthase gene $(d x s)$ were designed and denoted as the blaand $d x s$-set, respectively. The bla is a single-copy gene of pBR322 (Watson, 1988), and the $d x$ s is a singlecopy gene of $E$. coli chromosomal DNA (Hahn et al., 2001). Consequently, quantification of sample with the bla- and $d x s$-set represents corresponding amount of pBR322 and E. coli chromosomal DNA. The primer sets were designed using Primer3 software [Rozen and Skaletsky, 2000] based on the pBR322 sequence and the $E$. coli $d x s$ sequence. The sequences of the primers are shown in Table 1.

\subsection{Preparation of template DNA for real-time $Q P C R$}

The E. coli $\mathrm{DH} 5 \alpha$ transformant cells harboring pBR322 were cultured in two identical culture tubes (i.e., $50 \mathrm{ml}$ ). Total DNA was extracted from each of the cultures during the exponential growth phase, which was determined by periodic measurement of optical density (OD) at $600 \mathrm{~nm}$. The extraction was performed using the QIAamp DNA Mini kit (Qiagen), following a method for bacterial cells described in the manufacturer's instructions. The template DNA extracted from $E$. coli was normalized to $2 \mathrm{ng} / \mu \mathrm{l}$ with deionized and distilled water (DDW) for consistent QPCR assay. The concentration of extracted DNA was measured using aTD-700 fluorometer (Turner Designs) with the 
Table 1

Sequences of primers for real-lime QPCR

\begin{tabular}{|c|c|c|c|c|c|}
\hline Target & Accession no. & Primers $\left(5^{\prime} \rightarrow 3^{\prime}\right)^{\mathrm{a}}$ & Length (nt) & Primer position & Product size (bp) \\
\hline \multirow[t]{2}{*}{ bla } & J10749 & F: CTACGATACGGGAGGGCTTA & 20 & $3382-3401$ & 81 \\
\hline & & R: ATAAATCTGGAGCCGGTGAG & 20 & $3443-3462$ & \\
\hline \multirow[t]{2}{*}{$d x s$} & AF035440 & F: CGAGAAACTGGCGATCCTTA & 20 & $1509-1528$ & 113 \\
\hline & & R: CTTCATCAAGCGGTTTCACA & 20 & $1602-1621$ & \\
\hline
\end{tabular}

${ }^{a} \mathrm{~F}$ and $\mathrm{R}$ indicate forward and reverse primers, respectively.

PicoGreen dsDNA Quantification Reagent (Molecular Probes). The prepared template DNA was analyzed to quantify bla and $d x s$ in triplicate in real-time QPCR assay.

\subsection{Real-time QPCR using SYBR Green I dye}

Real-time QPCR amplification and analysis were performed using a LightCycler instrument with software version 3.5 (Roche Diagnostics). The threshold cycle $\left(C_{\mathrm{T}}\right)$ was determined by the 'Fit Points Method' in the software.

The QPCR assays with the sets were optimized with respect to the $\mathrm{MgCl}_{2}$ concentration and the annealing temperature (Yu et al., 2005). The optimal conditions were $3 \mathrm{mM} \mathrm{MgCl} 2$ and $62{ }^{\circ} \mathrm{C}$ for both primer sets detecting the bla and $d x s$ (data not shown). The realtime QPCR mixture of $20 \mu \mathrm{l}$ was prepared using the LightCycler FastStart DNA Master SYBR Green I kit (Roche Diagnostics): $12.4 \mu$ l PCR-grade water, $1.6 \mu \mathrm{l}$ $\mathrm{MgCl}_{2}$ stock solution (final concentration $3 \mathrm{mM}$ ), $1 \mu \mathrm{l}$ of each primer (final concentration $0.5 \mu \mathrm{M}$ ), $2 \mu \mathrm{l}$ reaction mix $10 \times$ solution, and $2 \mu l$ template DNA. The thermal cycling protocol was as follows: initial denaturation for 10 min at $95^{\circ} \mathrm{C}$ followed by 40 cycles of $10 \mathrm{~s}$ at $95^{\circ} \mathrm{C}, 10 \mathrm{~s}$ at $62^{\circ} \mathrm{C}$ and $10 \mathrm{~s}$ at $72^{\circ} \mathrm{C}$. The fluorescence signal was measured at the end of each extension step at $72{ }^{\circ} \mathrm{C}$. After the amplification, a melting curve analysis with a temperature gradient of $0.1{ }^{\circ} \mathrm{C} / \mathrm{s}$ from 70 to $95^{\circ} \mathrm{C}$ was performed to confirm that only the specific products were amplified. Finally, the samples were cooled down to $40^{\circ} \mathrm{C}$ for $30 \mathrm{~s}$.

SYBR Green I dye binds to double-stranded DNA in a sequence-independent way and emits fluorescence; thus specific and non-specific PCR products are both detected (Deprez et al., 2002). Therefore, amplification of non-specific PCR products must be avoided for exact quantification (Wilhelm et al., 2003). A melting curve analysis is frequently performed to rule out nonspecific PCR (Ririe et al., 1997). The melting process of double-stranded DNA causes a sharp reduction in the fluorescence signal around the melting temperature $\left(T_{\mathrm{m}}\right)$ of the PCR product, resulting in a clear peak in the negative derivative of the melting curve $(-\mathrm{d} F / \mathrm{d} T)$. Therefore, different fragments with different melting temperatures appear as separate peaks (Ririe et al., 1997).

\subsection{Construction of standard curves for plasmid copy number determination}

The PCR product of primers specific for $d x s$ of E. coli was amplified by conventional PCR using the $d x s$-set (Table 1). The reaction mixture of $50 \mu l$ contained $10 \mathrm{mM}$ Tris- $\mathrm{HCl}$ (pH 9.0), $40 \mathrm{mM} \mathrm{KCl}, 1.5 \mathrm{mM}$ $\mathrm{MgCl}_{2}, 250 \mu \mathrm{M}$ of each deoxynucleotide (dNTP), $0.5 \mathrm{mM}$ of each primer, $2.5 \mathrm{U}$ Taq DNA polymerase (Takara), and $50 \mathrm{ng}$ E. coli DH5 $\alpha$ genomic DNA template. PCR amplification was performed with a PTC100 thermal cycler (MJ research) according to the following program: an initial denaturation at $95^{\circ} \mathrm{C}$ for $5 \mathrm{~min}$, followed by 35 cycles of $30 \mathrm{~s}$ at $95^{\circ} \mathrm{C}, 30 \mathrm{~s}$ at $55^{\circ} \mathrm{C}$ and $1 \mathrm{~min}$ at $72^{\circ} \mathrm{C}$; a final extension at $72^{\circ} \mathrm{C}$ for $5 \mathrm{~min}$. The PCR product was purified using the GENECLEAN Turbo kit (Qbio gene) and cloned into a pGEM-T Easy vector (Promega). The cloned plasmid was purified using the QIAprep Spin Miniprep Kit (Qiagen). The resulting plasmid contained two separate sequences specific for bla and $d x s$ because the pGEM-T Easy vector contains a bla sequence identical with the bla in pBR322. Therefore, the plasmid can be detected by using either bla- or $d x s$-set. The plasmid was denoted as pGEM-BD.

A 10-fold serial dilution series of the pGEM-BD, ranging from $1 \times 10^{5}$ to $1 \times 10^{9} \mathrm{copies} / \mu \mathrm{l}$, was used to construct the standard curves for both bla and $d x s$. 
The concentration of the plasmid was measured using a fluorometer and the corresponding copy number was calculated using the following equation (Whelan et al., 2003):

DNA (copy)

$$
=\frac{6.02 \times 10^{23}(\text { copy } / \mathrm{mol}) \times \text { DNA amount }(\mathrm{g})}{\text { DNA length }(\mathrm{dp}) \times 660(\mathrm{~g} / \mathrm{mol} / \mathrm{dp})}
$$

$C_{\mathrm{T}}$ values in each dilution were measured in duplicate using a real-time QPCR with the bla- and $d x s$-sets to generate the standard curves for bla and $d x s$, respectively. The $C_{\mathrm{T}}$ values were plotted against the logarithm of their initial template copy numbers. Each standard curve was generated by a linear regression of the plotted points. From the slope of each standard curve, PCR amplification efficiency $(E)$ was calculated according to the equation (Rasmussen, 2001):

$E=10^{-1 / \text { slope }}-1$

\subsection{Absolute and relative quantification in real-time $Q P C R$}

Absolute and relative quantification methods (Klein, 2002) were simultaneously used to quantify the plasmid copy. Absolute quantification determines the exact copy concentration of target gene by relating the $C_{\mathrm{T}}$ value to a standard curve (Yu et al., 2005). Relative quantification presents the amount of target gene in a sample relative to another sample (i.e., a calibrator), which contains both target and reference genes with a constant ratio. The target/reference ratios of all samples are normalized by the target/reference ratio of the calibrator. PCR amplification efficiencies $(E)$ of target and reference sequences are required for the relative calculation (Lehmann and Kreipe, 2001; Roche, 2001). The results are expressed as a fold ratio of the normalized target amounts. In this experiment, bla and $d x s$ were used as the target and reference genes, respectively.

Relative quantification was performed by the $\Delta \Delta C_{\mathrm{T}}$ method (Livak and Schmittgen, 2001):

relative amount of target $=(1+E)^{-\Delta \Delta C_{\mathrm{T}}}$

where $\Delta \Delta C_{\mathrm{T}}: \Delta C_{\mathrm{T}}$ of the sample $-\Delta C_{\mathrm{T}}$ of the calibrator, $\Delta C_{\mathrm{T}}$ : $C_{\mathrm{T}}$ of the target $-C_{\mathrm{T}}$ of the reference, target: bla in pBR322, reference: $d x s$ in E. coli chro- mosomal DNA, sample: total DNA extracted from the transformed E. coli and calibrator: pGEM-BD.

\section{Results}

\subsection{Amplification specificity confirmation of primer sets}

The amplification specificity was checked by both melting curve analysis and gel electrophoresis. Fig. 1 shows the melting peaks and the corresponding gel band pictures for the real-time QPCR products with the bla-set (A) and the $d x s$-set (B). The bla-set showed a sharp peak for the PCR product of the quantitative standard sample, pGEM-BD, at $87.0^{\circ} \mathrm{C}$. A single melting peak at the same melting temperature was produced for the PCR product of the total DNA sample. The $d x s$-set showed a single melting peak for the quantitative standard as well as for the total DNA sample, at the same temperature of $86.5^{\circ} \mathrm{C}$. Every PCR product also generated prominent bands with expected sizes in the gel electrophoresis analysis. The identity of amplified products was additionally confirmed by sequencing analysis (Fig. 1). These results indicated that non-specific PCR products with the primer sets were not detected in the analyzed temperature range.

\subsection{Standard curves and amplification efficiencies}

The standard curves for bla and $d x s$, each ranging from $1 \times 10^{5}$ to $1 \times 10^{9} \mathrm{copies} / \mu \mathrm{l}$, are shown in Fig. 2A. Both curves were linear in the range tested $\left(R^{2}>0.999\right)$ by the duplicate reactions. The slopes of the standard curves for bla and $d x s$ were -3.42 and -3.43 , respectively, which were identical (Nystorm et al., 2004). From the slopes, a high amplification efficiency of 0.96 was determined for both bla and $d x s$ in the investigated range and was used for relative quantification.

\subsection{Validation of the $\Delta \Delta C_{T}$ method}

For the $\Delta \Delta C_{\mathrm{T}}$ calculation to be valid, the amplification efficiencies of the target and reference must be approximately equal $\left(E_{\text {target }}=E_{\text {reference }}=E\right)$. The variation of $\Delta C_{\mathrm{T}}$, the $C_{\mathrm{T}}$ deviation between bla and $d x s$, with template dilution was examined to validate the 


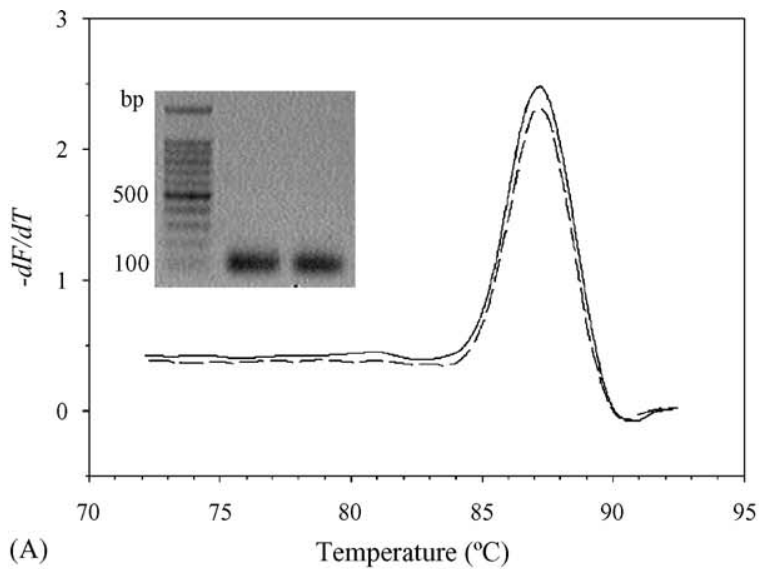

bla PCR product from standrard sample:bla PCR product from E.coli total DNA Identity $=100.00 \%(81 / 81)$ gap $=0.00 \%(0 / 81)$

1 CTACGATACG GGagGCTTA CCATCTGGCC CCAGTGCTGC AATGATACCG CGAGACCCAC

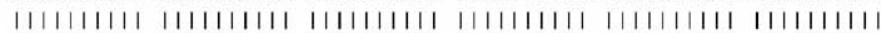

1 CTACGATACG GGAGGGCTTA CCATCTGGCC CCAGTGCTGC AATGATACCG CGAGACCCAC

61 GCTCACCGGC TCCAGATTTA T

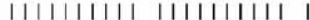

61 GCTCACCGGC TCCHGATTTA T

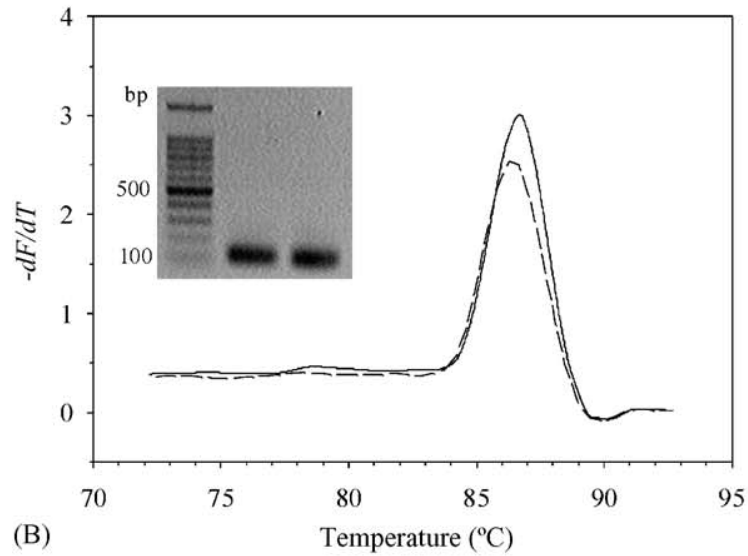

dxs PCR product from standrard sample:dxs PCR product from E.coli total DNA Identity $=100.00 \div(113 / 113)$ gap $=0.00 \%(0 / 113)$

1

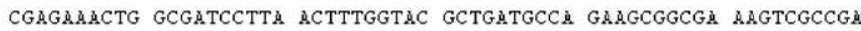

|||||||||||||||||||||||||||||||||||||||||||||||||||||||||| $\mid$

1 CGAGAAATG GCGATCCTTA ACTTTGGTAC GCTGATGCCA GAAGGGCGA AAGTCGCCG

61 atCGCTGAAC GCCACGCTGG TCGATATGCG TTTTGTGAM CCGCTTGATG AAG

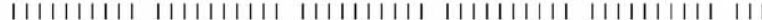

61 ATCGCTGAAC GCCACGCTGG TCGATATGCG TTTTGTGAA CCGCTTGATG AAG

Fig. 1. Confirmation of PCR amplification specificities of bla-set (A) and the $d x s$-set (B). Melting peaks were examined for the bla- and the $d x s$-sets with a quantitative standard sample (solid line) and an E. coli total DNA sample (dashed line) as templates. Gel electrophoresis of the PCR products was performed on $1 \%$ agarose gel. The melting temperatures and the amplicon sizes were $87.0^{\circ} \mathrm{C}$ and $81 \mathrm{bp}$ for the bla-set, and $86.5^{\circ} \mathrm{C}$ and $113 \mathrm{bp}$ for the $d x s$-set. For each set, the identity of the amplified products was confirmed by DNA sequencing analysis. 


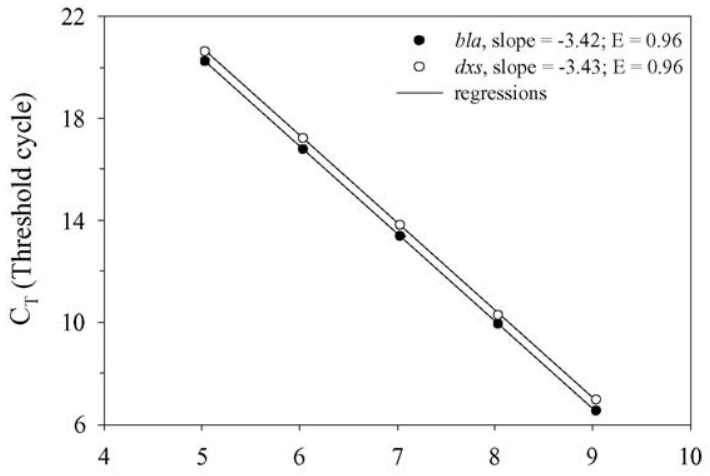

(A)

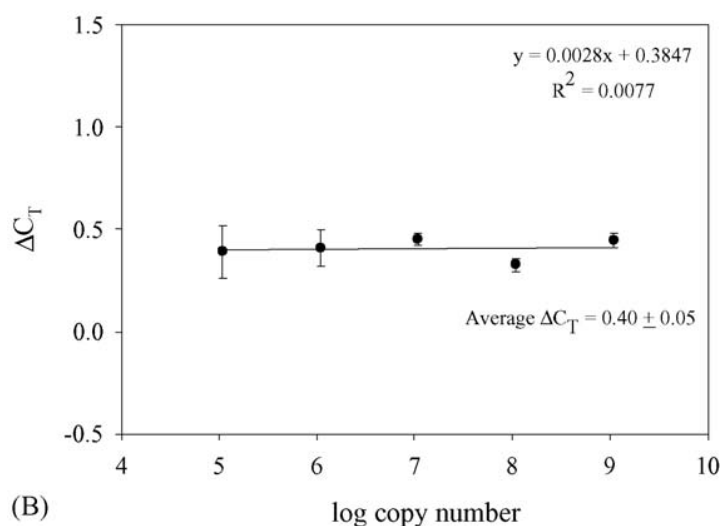

Fig. 2. Construction of the standard curves for bla and $d x s$ (A) and validation of the $\Delta \Delta C_{\mathrm{T}}$ calculation (B). The standard curves were constructed with serial 10-fold dilutions of the pGEM-BD, ranging from $1 \times 10^{5}$ to $1 \times 10^{9}$ copies/ $\mu$ l. Each standard dilution was amplified by real-time QPCR using bla- and $d x s$-set in duplicate. For each gene, determined $C_{\mathrm{T}}$ values were plotted against the logarithm of their known initial copy numbers $(n=2)$. A standard curve was generated by linear regression through these points (A). The $\Delta C_{\mathrm{T}}$, the $C_{\mathrm{T}}$ deviation of bla vs. $d x s$, was calculated for each dilution and plotted $(n=2)(\mathrm{B})$. Average $\Delta C_{\mathrm{T}}=$ average \pm S.D. $(n=10)$.
$\Delta \Delta C_{\mathrm{T}}$ method. The $\Delta C_{\mathrm{T}}$ was calculated using each of the 10 -fold serial dilutions of the pGEM-BD. A plot of the logarithm of initial copy number versus $\Delta C_{\mathrm{T}}$ was made (Fig. 2B) and the slope of the regression line was close to zero (0.0028). This indicates that the amplification efficiencies of bla and $d x s$ are identical enough for the $\Delta \Delta C_{\mathrm{T}}$ calculation (Livak and Schmittgen, 2001).

\subsection{Determination of plasmid copy number}

Real-time QPCR amplifications of the total DNA samples from two separate cultures of E. coli DH5 $\alpha$ harboring pBR322 and standard samples were performed simultaneously. Absolute quantification was performed using the standard curves constructed (Fig. 2A). The absolute copy numbers of bla and $d x s$ in the $E$. coli total DNA samples were determined from the corresponding standard curves, using the $C_{\mathrm{T}}$ values. The plasmid copy number of pBR322 was then calculated by dividing the copy number of bla by the copy number of $d x s$. Because bla and $d x s$ are singlecopy genes of pBR322 and E. coli chromosomal DNA, respectively, the ratio of bla to $d x s$ is equal to the plasmid copy number of pBR322. The results from the absolute quantification and the calculated plasmid copy numbers are shown in Table 2. The total DNA samples from the two separate cultures showed similar plasmid copy numbers of 18.7 and 18.3 .

For relative quantification, bla and $d x s$ were used as the target and the reference genes, respectively. Because pGEM-BD (i.e., calibrator) contained one copy of each $d x s$ and bla specific sequence, the copy ratio of bla to $d x s$ in the calibrator is also 1 . Therefore, the result from the $\Delta \Delta C_{\mathrm{T}}$ calculation is equal to the copy ratio of bla to $d x s$, i.e., the plasmid copy number of pBR322 in the sample. The $\Delta C_{\mathrm{T}}$ of the calibrator was given as $0.40 \pm 0.05$ by averaging the

Table 2

Estimated plasmid copy number (PCN) by absolute quantification

\begin{tabular}{|c|c|c|c|c|c|}
\hline \multirow[t]{2}{*}{ Culture } & \multicolumn{2}{|l|}{$C_{\mathrm{T}^{\mathrm{a}}}$} & \multicolumn{2}{|l|}{ Copies $^{\mathrm{b}}($ copies/ $\mu \mathrm{l})$} & \multirow[t]{2}{*}{$\mathrm{PCN}^{\mathrm{c}}$} \\
\hline & bla & $d x s$ & bla & $d x s$ & \\
\hline 1 & $13.73 \pm 0.03$ & $17.68 \pm 0.05$ & $1.12 \times 10^{7}(2.0 \%)$ & $5.98 \times 10^{5}(3.4 \%)$ & $18.7(3.9 \%)$ \\
\hline 2 & $13.86 \pm 0.03$ & $17.78 \pm 0.04$ & $1.03 \times 10^{7}(2.1 \%)$ & $5.61 \times 10^{5}(2.8 \%)$ & $18.3(3.5 \%)$ \\
\hline
\end{tabular}

\footnotetext{
a Average \pm S.D. $(n=3)$.

b Average (coefficient of variation) $(n=3)$.

c Average (coefficient of variation) $(n=3)$.
} 
Table 3

Estimated plasmid copy number $(\mathrm{PCN})$ by relative quantification

\begin{tabular}{llllll}
\hline Culture & $\Delta C_{\mathrm{T}}$ sample $^{\mathrm{a}}$ & Calibrator $^{\mathrm{b}}$ & $\Delta \Delta C_{\mathrm{T}}^{\mathrm{c}}$ & $\mathrm{PCN}^{\mathrm{d}}$ \\
\hline 1 & & & $2^{-\Delta \Delta C_{\mathrm{T}}}$ \\
\hline & $-3.95 \pm 0.06$ & $0.40 \pm 0.05$ & $-4.35 \pm 0.08$ & $20.5(5.4 \%)$ & $1.96^{-\Delta \Delta C_{\mathrm{T}}}$ \\
\hline
\end{tabular}

a Average \pm S.D. $(n=3)$.

${ }^{\mathrm{b}}$ Calculated from the serial dilutions of the quantitative standard sample used for standard curve construction. Average \pm S.D. $(n=10)$.

c Average \pm S.D. $(n=3)$.

d Average (coefficient of variation) $(n=3)$.

$\Delta C_{\mathrm{T}}$ values determined from the 10 -fold serial dilutions of the pGEM-BD (Fig. 2B). The determination of $\Delta C_{\mathrm{T}}$ of each dilution was performed in duplicate. The results from the relative quantification and the calculated plasmid copy numbers are shown in Table 3. Based on Eq. (3) with the assumption that the amplification efficiency $(E)$ is 1 , the plasmid copy number was determined by the $2^{-\Delta \Delta C_{\mathrm{T}}}$ calculation. The results were 20.5 and 20.0 for the total DNA samples from the separate $E$. coli cultures. Using the experimentally calculated value (Fig. 2A) of 0.96 for $E$ in Eq. (3), the plasmid copy numbers were 18.7 and 18.4 for each sample. This calculation was also valid because the amplification efficiencies of bla and $d x s$ were almost identical to each other (Fig. 2).

The results from both absolute and relative quantification were reproducible and close to the reported values. The plasmid copy number of pBR322 is known to be 15-20 within E. coli host cells (Muesing et al., 1981; Lin-Chao and Bremer, 1986; Qiagen, 2004). The coefficient of variation (CV) values calculated from the absolute and relative quantification results were $2.8-3.9 \%$ and $4.7-5.4 \%$, respectively. These values are about half the $C V$ from a recent report on the development of a new plasmid copy number assay method, 7.1-9.8\% (Pushnova et al., 2000).

\section{Discussion}

We demonstrated the feasibility of two real-time QPCR methods to determine plasmid copy number. The absolute and relative quantification methods require the separate detection of the plasmid and the host chromosomal DNA. The detection is performed using a primer set specific to a single-copy gene in each target molecule.
In this study, SYBR Green I dye was used in realtime QPCR analysis because it has several advantages over sequence-specific probes when singleplex PCRs are performed. Both detection formats have about the same detection limit, reproducibility, and dynamic range (Deprez et al., 2002; Wilhelm et al., 2003). The accumulation of primer dimers and the amplification of non-specific PCR products can be analyzed only in SYBR Green I detection (Deprez et al., 2002). In a previous study, SYBR Green I detection was used to determine host DNA contamination in plasmid preparations and provided a reliable alternative to traditional blotting methods and to more expensive TaqMan protocols (Vilalta et al., 2002).

We applied the real-time QPCR based methods to quantify the plasmid copy number of pBR322 within E. coli DH5 $\alpha$ host cells. The results corresponded to the previously known values and were precise with $\mathrm{CV}$ values of $2.8-5.4 \%$ (Tables 2 and 3). It should be noted that the relative quantification results were nearly equal to the absolute quantification results when the calculated value of amplification efficiency $(E), 0.96$, was used for the $(1+E)^{-\Delta \Delta C_{\mathrm{T}}}$ calculation. This agreement means that the relative quantification based method established in this study was successfully applied to the determination of plasmid copy number in the DNA.

For reliable analysis, stable storage of quantitative standards is very important. We used the recombinant plasmid pGEM-BD as a standard sample. Use of cloned targets in carrier DNA is common in real-time QPCR analyses (Whelan et al., 2003).

Two real-time QPCR methods (i.e., absolute and relative) with the primer sets designed in this study and the pGEM-BD can be directly applied to any recombinant system which uses a plasmid containing bla with E. coli as the host cell. The ampicillin resistance gene, bla is one of the most frequently used genetic markers 
for recombinant plasmid, and E. coli is the most popular host cell for bacterial recombination. Therefore, the present technique should be widely applicable.

\section{Acknowledgement}

This research was supported in part by the Korea Energy Management Corporation and Advanced Environmental Biotechnology Research Center (AEBRC) (Grant No. R11-2003-006-02002-0) program.

\section{References}

Argyropoulos, D., Savva, D., 1997. A fast fluorescence method for accurate determination of plasmid copy number in Bacillus subtilis. Biotechnol. Tech. 11, 605-609.

Burgos, J.S., Ramirez, C., Tenorio, R., Sastre, I., Bullido, M.J., 2002. Influence of reagents formulation on real-time PCR parameters. Mol. Cell. Probe 16, 257-260.

Deprez, R.H.L., Fijnvandraat, A.C., Ruijter, J.M., Moorman, A.F.M., 2002. Sensitivity and accuracy of quantitative real-time polymerase chain reaction using SYBR green I depends on cDNA synthesis conditions. Anal. Biochem. 307, 63-69.

Ferre, F., 1992. Quantitative or semi-quantitative PCR: reality versus myth. PCR Meth. Appl. 2, 1-9.

Fujimura, H., Sakuma, Y., Weimer, T., 1996. A quantitative method for the determination of plasmid copy number in recombinant yeast. Biologicals 24, 67-70.

Hahn, K., Eubanks, L.M., Testa, C.A., Blagg, B.J., Baker, J.A., Poulter, C.D., 2001. 1-deoxy-D-xylulose 5-phosphate synthase, the gene product of open reading frame (ORF) 2816 and ORF 2895 in Rhodobacter capsulatus. J. Bacteriol. 183, 1-11.

Klein, D., 2002. Quantification using real-time PCR technology: applications and limitations. Trends Mol. Med. 8, 257-260.

Korpela, K., Buchert, P., Soderlund, H., 1987. Determination of plasmid copy number with nucleic acid sandwich hybridization. J. Biotechnol. 5, 267-277.

Lehmann, U., Kreipe, H., 2001. Real-time PCR analysis of DNA and RNA extracted from formalin-fixed and paraffin-embedded biopsies. Methods 25, 409-418.

Lin-Chao, S., Bremer, H., 1986. Effect of the bacterial growth rate on replication control of plasmid pBR322 in Escherichia coli. Mol. Gen. Genet. 203, 143-149.
Livak, K.J., Schmittgen, T.D., 2001. Analysis of relative gene expression data using real time quantitative PCR and the $2^{-\Delta \Delta C_{\mathrm{T}}}$ method. Methods 25, 402-408.

Muesing, M., Tamm, J., Shepard, H.M., Polisky, B., 1981. A single base-pair alteration is responsible for the DNA overproduction phenotype of a plasmid copy-number mutant. Cell 24, 235242.

Nystorm, K., Biller, M., Grahn, A., Lindh, M., Larson, G., Olofsson, S., 2004. Real time PCR for monitoring regulation of host gene expression in herpes simplex virus type 1-infected human dipoild cells. J. Virol. Meth. 118, 83-94.

Pushnova, E.A., Geier, M., Zhu, Y.S., 2000. An easy and accurate agarose gel assay for quantitation of bacterial plasmid copy numbers. Anal. Biochem. 284, 70-76.

Qiagen, 2004. Origin of replication and copy number of various plasmids and cosmids. Qiagen Literature Resoreces. http://www1.qiagen.com/resources/info/plasmid_specifications. aspx\#1.

Rasmussen, R., 2001. In: Meuer, S., Wittwer, C., Nakagawara, K. (Eds.), Quantification on the LightCycler. Springer-Verlag, Berlin, Germany, pp. 21-34.

Ririe, K.M., Rasmussen, R.P., Wittwer, C.T., 1997. Product differentiation by analysis of DNA melting curves during the polymerase chain reaction. Anal. Biochem. 245, 154-160.

Roche, 2001. LightCycler: Relative Quantification. Technical Note, LC13/2001, pp. 1-28.

Rozen, S., Skaletsky, H., 2000. Primer 3 on the WWW for general users and for biologist programmers. Methods Mol. Biol. 132, $365-386$.

Summers, D.K., 1991. The kinetics of plasmid loss. Trends Biotechnol. 9, 273-278.

Vilalta, A., Whitlow, V., Martin, T., 2002. Real-time PCR determination of Escherichia coli genomic DNA contamination in plasmid preparations. Anal. Biochem. 301, 151-153.

Watson, N., 1988. Anew revision of the sequence of plasmid pBR322. Gene 70, 399-403.

Whelan, J.A., Russel, N.B., Whelan, M.A., 2003. A method for the absolute quantification of cDNA using real time PCR. J. Immunol. Meth. 278, 261-269.

Wilhelm, J., Pingoud, A., Hahn, M., 2003. Real-time PCR-based method for the estimation of genome sizes. Nucl. Acids Res. 31, e56.

Yu, Y., Lee, C., Kim, J., Hwang, S., 2005. Group-specific primer and probe sets to detect methanogenic communities using quantitative real-time polymerase chain reaction. Biotechnol. Bioeng. 89, 670-679. 